\title{
Towards the True prediction of EHL Friction
}

\author{
M. Björling a,*, W. Habchi ${ }^{\mathrm{b}}$, S. Bair ${ }^{\mathrm{c}}$, R. Larsson ${ }^{\mathrm{a}}$, P. Marklund ${ }^{\mathrm{a}}$ \\ ${ }^{a}$ Division of Machine Elements, Department of Engineering Science and Mathematics, Luleå University of Technology, Luleå, SE-97187 Sweden \\ ${ }^{b}$ Lebanese American University, Department of Industrial and Mechanical Engineering, Byblos, Lebanon \\ ${ }^{c}$ G.W. Woodruff School of Mechanical Engineering, Centre for High Pressure Rheology, Georgia Institute of Technology, Atlanta, GA 30332-0405, USA
}

\begin{abstract}
The capability to predict elastohydrodynamic film-thickness and friction from primary measurements of transport properties of liquid has been an elusive goal for tribologists for 50 years. Most comparisons between predictions and experiments involve some amount of tuning of the model in order to match the experimental results. In true prediction, this cannot be done since there are normally no experimental results to compare to. Primary measurements of lubricant transport properties of Squalane were performed, and used in a numerical friction prediction model. Afterwards, friction was measured in a ball-on-disc tribotester. No tuning of the lubricant properties, model or test setup were applied. The current work on EHL-friction is therefore a true representation of the current level of EHL-friction prediction.
\end{abstract}

Keywords: Elastohydrodynamic, Simulation, Friction, Thermal-effects

\section{Introduction}

Elastohydrodynamic lubrication (EHL) is said to occur in lubricated, non-conformal contacts where the elastic deformation of the mating surfaces has a significant influence on the lubricant film thickness. In many cases the elastic deformation occurs even for materials with a high young's modulus due to high contact pressures. These high pressures will in general give rise to a substantial viscosity increase in the lubricant. Machine components like gears, rolling element bearings and cam followers operate under EHL conditions. Improving the performance of machine components working under EHL regime is of great importance to reduce wear and increase efficiency.

The capability to predict elastohydrodynamic film thickness and friction from primary measurements of the transport properties of the liquid has been an elusive goal of the tribology community for more than fifty years. Indeed, such a capability may be considered an indication of the maturity of the EHL field and of the understanding it has provided of the mechanics governing the lubrication process. Nearly all of the research in EHL has involved the assumption of simple but unrealistic rheological models and the adjustment of the parameters of these models for validation. Clearly, a complete understanding of the film forming process, free of adjustable properties, must precede a prediction of friction since the film thickness establishes the shear rate in the Hertz region where friction is mostly generated. It is important to keep in mind that the pressure range, and thus the viscosity increase inside the contact, that governs the coefficient of friction is several orders of magnitude higher than the pressures in the inlet that are governing film formation.

\footnotetext{
${ }^{*}$ Corresponding Author

Email address: marcus.bjorling@ltu.se (M. Björling)
}

The foundation for the prediction of film thickness and friction, at least for low pressures, has been available for some time. The pressure dependence of density, viscosity and thermal conductivity was accurately described to EHL pressures (1.2 GPa) by Bridgman [1] in 1931. Precise measurements of the temperature and pressure dependence of lubricants to EHL pressures were obtained in Bridgman's laboratory and reported in the 1953 ASME viscosity report [2]. The nature of non-Newtonian response to shear stress in lubricants was correctly described by Hutton in 1973 [3]. Therefore, much of the needed rheological descriptions have been in place for about four decades and all that has been required has been to perform the computations with accurately measured properties. Some good predictions have been obtained for squalane and a polyalphaolefin using simple isothermal calculations where the film thickness was assumed uniform over the Hertz region resulting in the Hertz pressure distribution $[4,5]$. The analysis for the polyalphaolefin was improved by a full EHL simulation [6] which calculated the real distribution of film thickness and pressure, resulting in an extraordinarily precise agreement with measured contact behavior although at low pressure and sliding velocity.

Successful predictions in the high pressure, thermal regime have come more recently. Using the "full system approach" [7, 8], it has been possible to predict EHL friction [9] accurately at very high contact pressure with significant sliding. The only property which was not obtained from a primary measurement was the limiting-stress pressure coefficient. The ability to measure shear stress in a liquid when the response is rateindependent [10] apparently has been lost for about twenty years. However, when the other rheological properties are wellknown an approximation of this coefficient may be extracted from a traction measurement with relative confidence. It should be pointed out that recent reseach [11] has shown that an accu- 


\begin{tabular}{|c|c|c|c|}
\hline \multicolumn{4}{|c|}{ Nomenclature } \\
\hline$\beta_{K}$ & Temperature coefficient of $K_{0}\left[K^{-1}\right]$ & $G$ & Effective shear modulus $[\mathrm{Pa}]$ \\
\hline$\chi$ & Dimensionless heat capacity scaling parameter & $g$ & Thermodynamic interaction parameter \\
\hline$\eta$ & Generalized (shear dependent) viscosity $[\mathrm{Pas}]$ & $k$ & Thermal conductivity $[\mathrm{W} / \mathrm{mK}]$ \\
\hline$\gamma$ & Shear rate $\left[s^{-} 1\right]$ & \multirow[t]{2}{*}{$K_{0}^{\prime}$} & \multirow{2}{*}{$\begin{array}{l}\text { Pressure rate of change of isothermal bulk modulus } \\
\text { at } p=0\end{array}$} \\
\hline$\kappa$ & Dimensionless conductivity scaling parameter & & \\
\hline$\Lambda$ & Limiting stress pressure coefficient & $K_{00}$ & $K_{0}$ at zero absolute temperature $[\mathrm{Pa}]$ \\
\hline$\lambda_{r}$ & Relaxation time at $T_{R}$ and ambient pressure $[s]$ & $K_{0}$ & Isothermal bulk modulus at $p=0[\mathrm{~Pa}]$ \\
\hline$\mu$ & Limiting low-shear viscosity $[\mathrm{Pas}]$ & $L$ & Contact load $[\mathrm{N}]$ \\
\hline$\mu_{R}$ & Low shear viscosity at $T_{R}$ and ambient pressure $[\mathrm{Pas}]$ & $m$ & Parameter in the heat capacity function $\left[\mathrm{J} / \mathrm{m}^{3} \mathrm{~K}\right]$ \\
\hline$\mu_{\infty}$ & Viscosity extrapolated to infinite temperature $[P a s]$ & $n$ & Power law exponent \\
\hline$\rho$ & Lubricants density $[\mathrm{kg}]$ & $p$ & Pressure $[\mathrm{Pa}]$ \\
\hline$\tau$ & Shear stress $[\mathrm{Pa}]$ & \multirow[t]{2}{*}{$q$} & \multirow{2}{*}{$\begin{array}{l}\text { Coefficient in the dimensionless conductivity scaling } \\
\text { parameter }\end{array}$} \\
\hline$\varphi$ & Dimensionless viscosity scaling parameter & & \\
\hline$\varphi_{\infty}$ & Viscosity scaling parameter for unbounded viscosity & $s$ & Exponent in the conductivity scaling model \\
\hline$A$ & Coefficient in the dimensionless conductivity scaling & $S R R$ & Slide to roll ratio \\
\hline \multirow[b]{2}{*}{$a_{v}$} & \multirow{2}{*}{$\begin{array}{l}\text { Thermal expansivity defined for volume linear with } \\
\text { temperature }\left[K^{-1}\right]\end{array}$} & $T$ & Temperature $[\mathrm{K}]$ \\
\hline & & $T_{R}$ & \\
\hline$B_{F}$ & Fragility parameter in the new viscosity equation & $U_{e}$ & Mean entrainment speed $[\mathrm{m} / \mathrm{s}]$ \\
\hline$C$ & Parameter in the conductivity function $[\mathrm{W} / \mathrm{mK}]$ & $V$ & Volume $\left[m^{3}\right]$ \\
\hline$C_{0}$ & Parameter in the heat capacity function $\left[\mathrm{J} / \mathrm{m}^{3} \mathrm{~K}\right]$ & $V_{0}$ & Volume at $\mathrm{p}=0\left[\mathrm{~m}^{3}\right]$ \\
\hline$c_{p}$ & Specific heat capacity $[J / k g K]$ & $V_{R}$ & Volume at reference state, $T_{R}, \mathrm{p}=0\left[\mathrm{~m}^{3}\right]$ \\
\hline$C_{v}$ & Lubricants volumetric heat capacity & $W_{f}$ & Friction power $[\mathrm{W}]$ \\
\hline
\end{tabular}

rate value of limiting shear stress cannot be obtained from a traction measurement unless the viscosity is known to be very large. This is because the magnitude of the friction cofficient in the top parts of a friction vs slip curve is not only governed by the limiting shear stress, but also shear-thinning.

Most comparisons between predictions and experiments involve some amount of tuning of the model in order to match the experimental results. In real true prediction this cannot be done since there are normally no experimental results to compare to. Instead, all three components occur independently from each other:

- Primary measurement of lubricant transport properties.

- Prediction using measured transport properties as input data together with operation and geometric data.

- Experimental validation

Such an investigation is presented here. The three components in this work have been carried out at three different laboratories. Initially the transport properties of squalane (a low molecular weight branched alkane) were characterized at Georgia Tech. These properties were later used in a numerical model to predict friction at the Lebanese American University. Finally friction was measured in a ball on disc test device at Luleå University of Technology. No tuning of the lubricant properties, model and test setup has been applied. The presented results on EHL friction are therefore a true representation of the current level of EHL friction prediction. This study also includes higher entrainment speeds than previously published in this kind of investigation.

\section{Overall Methodology}

The following sections cover the investigated cases, including running conditions and loads. It also contains information about the lubricant and its transport properties, as well as the numerical model and the underlying boundary conditions and assumptions. Finally, the experimental equipment and specimens are discussed together with the test procedure. 


\begin{tabular}{lc}
\multicolumn{2}{c}{ Table 1: Investigated conditions } \\
\hline Temperature & $40{ }^{\circ} \mathrm{C}$ \\
Contact load & 50 and $300 \mathrm{~N}$ \\
Maximum hertzian pressure & 1.07 and $1.94 \mathrm{GPa}$ \\
Entrainment speed, $U_{e}$ & 0.34 to $9.6 \mathrm{~m} / \mathrm{s}$ \\
Slide to Roll Ratio, SRR & 0.0002 to 0.49 \\
\hline
\end{tabular}

\subsection{Investigation Procedure}

The numerical prediction and experimental measurements are performed using the same conditions reported in Table 1. SRR is defined as the speed difference divided by the mean entrainment speed, $U_{e}$. All tests are performed with positive sliding only, which in this case means that the ball is rotating faster than the disc. The investigation includes a combination of high entrainment speeds and contact pressures that to the authors knowledge has not been considered in any similar previous studies.

Both numerical predictions and experiments were performed with the same lubricant, squalane, a commercially available low molecular weight branched alkane $(2,6,10,15,19,23$ hexamethyltetracosane). An oil without additives was chosen to minimize the effect of tribochemical reactions on the friction coefficient. As explained later the tests are conducted repeatedly until a satisfactory repeatability is reached, and since this could take a different amount of cycles for different test cases a lubricant containing additives could be in different stages of tribofilm development. At the test temperature of $40^{\circ} \mathrm{C}$, the ambient viscosity of squalane is $15 \mathrm{mPas}$ and the pressure viscosity coefficient is $18 \mathrm{GPa}^{-1}$ [12]. The following section gives a more in depth view of the lubricant parameters used in the numerical model.

\subsection{Lubricant transport properties}

The transport properties used for the numerical model in this study are obtained from several earlier studies on squalane. A brief overview of the models derived from these studies is given here. Further information about the measurements for the effect of pressure, temperature and shear on viscosity is found in references: $[4,12,13]$.

\subsubsection{Equation of state}

A temperature modified version of the Tait equation of state is used to model the temperature and pressure dependence of volume for Squalane. The Tait equation is written for the volume relative to the volume at ambient pressure,

$$
\frac{V}{V_{0}}=1-\frac{1}{1+K_{0}^{\prime}} \ln \left[1+\frac{p}{K_{0}}\left(1+K_{0}^{\prime}\right)\right]
$$

with

$$
K_{0}=K_{00} \exp \left(-\beta_{K} T\right)
$$

The volume at ambient pressure relative to the ambient pressure volume at the reference temperature, $T_{R}$, is assumed to vary with temperature as:

$$
\frac{V_{0}}{V_{R}}=1+a_{v}\left(T-T_{R}\right)
$$

where $K_{0}^{\prime}=11.74, a_{v}=8.36 \times 10^{-4} K^{-1}, K_{00}=8.658 \mathrm{GPa}$ and $\beta_{K}=6.332 \times 10^{-3} K^{-1}$ were obtained from experimental measurements with a standard deviation of $0.05 \%$ [12].

\subsubsection{Viscosity}

A thermodynamic scaling rule that has been found to be accurate for many organic liquids is: $\mu=f\left(T V^{g}\right)$, where $-3 \mathrm{~g}$ is related to the exponent of the repulsive intermolecular potential. A useful scaling parameter can therefore be written as:

$$
\varphi=\left(\frac{T}{T_{R}}\right)\left(\frac{V}{V_{R}}\right)^{g}
$$

An accurate scaling function can be obtained from a Vogel like form:

$$
\mu=\mu_{\infty} \exp \left(\frac{B_{F} \varphi_{\infty}}{\varphi-\varphi_{\infty}}\right)
$$

where $g=3.921, \varphi_{\infty}=0.1743, B_{F}=24.50$ and $\mu_{\infty}=0.9506 \times 10^{-4}$ $\mathrm{Pa} \cdot \mathrm{s}$ were obtained from experimental measurements with standard deviation of $14.9 \%$ with respect to relative viscosity [12].

For the shear dependence of viscosity, a t-T-p shifted Carreau equation is used:

$$
\eta(\gamma, p)=\mu\left[1+\left(\gamma \lambda_{R} \frac{\mu}{\mu_{R}} \frac{T}{T_{R}} \frac{V_{0}}{V_{R}}\right)^{2}\right]^{(n-1) / 2}
$$

where $\mu_{R}=15.6 \mathrm{mPa} \cdot \mathrm{s}, \gamma_{R}=2.26 \times 10^{-9} \mathrm{~s}$, and $\mathrm{n}=0.463$ were obtained from Non-Equilibrium Molecular Dynamics and experimental measurements [13].

The limiting shear stress was shown to depend on pressure as:

$$
\tau=\Lambda p
$$

where $\Lambda=0.075$ was found from EHL traction experiments and is assumed to be independent of temperature [4].

\subsubsection{Thermal properties}

The thermal conductivity and volumetric heat capacity of squalane is expressed as:

$$
k=C \kappa^{-s}
$$

with

$$
\kappa=\left(\frac{V}{V_{R}}\right)\left[1+A\left(\frac{T}{T_{R}}\right)\left(\frac{V}{V_{R}}\right)^{q}\right]
$$

And

$$
C_{v}=\rho c_{p}=C_{0}+m \chi
$$

with

$$
\chi=\left(\frac{T}{T_{R}}\right)\left(\frac{V}{V_{R}}\right)^{-3}
$$




\begin{tabular}{lc}
\multicolumn{2}{c}{ Table 2: Specimen material properties } \\
\hline Material & $52100(\mathrm{AISI})$ \\
Young's modulus $(\mathrm{Pa})$ & $210 \times 10^{9}$ \\
Poisson's coefficient & 0.3 \\
Specific heat capacity $(\mathrm{J} / \mathrm{kg} \mathrm{K})$ & 475 \\
Thermal conductivity $(\mathrm{W} / \mathrm{m} \mathrm{K})$ & 46.6 \\
Thermal diffusivity $\left(\mathrm{m}^{2} / \mathrm{s}\right)$ & $13.6 \times 10^{-6}$ \\
Density $\left(\mathrm{kg} / \mathrm{m}^{3}\right)$ & 7850 \\
\hline
\end{tabular}

where $C_{k}=0.074 \mathrm{~W} / \mathrm{m} \cdot \mathrm{K}, \mathrm{s}=4.5, C_{0}=0.94 \times 10^{6} \mathrm{~J} / \mathrm{m}^{3} \cdot \mathrm{K}$ and $\mathrm{m}=0.62 \times 10^{6} \mathrm{~J} / \mathrm{m}^{3} \cdot \mathrm{K}$ were obtained from experimental measurements. The thermal properties were measured by Ove Andersson at Umeå University.

\subsection{Numerical model}

The numerical model employed in this work was described in detail in $[8,9,14]$. In this section, only the main features of this model are recalled. The model is based on a finite element fully coupled resolution of the EHD equations: Reynolds, linear elasticity and load balance equations. The latter are solved simultaneously providing robust and fast converging solutions. The generalized Reynolds equation [15] is used to account for the shear dependence of the lubricant. Special formulations are introduced in order to stabilize the solution of Reynolds equation at high loads. The temperature distribution in the contact is obtained by solving the $3 \mathrm{D}$ energy equation in the lubricant film and solid bodies. The model incorporates the variations of the lubricant's thermal properties with pressure and temperature throughout the contact. Then an iterative procedure is applied between the respective solutions of the EHD and thermal problems as described in [8, 14]. During the iterative procedure, every time the shear stress $\tau$ is evaluated (using viscosity data provided by a combination of the Carreau and Vogel-like models) it is either truncated to $\tau_{L}$ if it exceeds $\tau_{L}$ or, otherwise, it is kept unchanged.

\subsection{Ball on disc tribotester}

The experiments were carried out with a Wedeven Associates Machine (WAM) 11, ball on disc test device. The lubricant is supplied at the center of the disc in an oil dispenser that distributes the lubricant across the disc surface. Lubricant is circulated in a closed loop from the oil bath, through a hose pump to the oil dispenser at the center of the disc. The hose pump is delivering approximately $180 \mathrm{ml} / \mathrm{min}$. Three thermocouples are used in the test setup, one located in the oil bath, one in the outlet of the oil supply and one trailing in the oil film close to the inlet region of the ball on disc contact. A more thorough description of the test rig and its features is presented in previous work [16].

\subsubsection{Test specimens}

All specimens used in the tests (balls and discs) are made from AISI 52100 bearing steel. The balls are grade 20 with a $13 / 16$ inch $(20.637 \mathrm{~mm})$ outer diameter and a hardness of about $60 \mathrm{HRC}$. The discs have a 4 inch $(101.6 \mathrm{~mm})$ outer diameter, a circumferential grind (before polish) and are through hardened to about $60 \mathrm{HRC}$. Additional material parameters also used in the numerical model are found in Table 2. The surface roughness, RMS, has been measured to about $25 \mathrm{~nm}$ for the balls, and $35 \mathrm{~nm}$ for the disc, which gives a combined roughness of approximately $43 \mathrm{~nm}$. The surface roughness measurements have been conducted in a Wyko NT1100 optical profilometer system from Veeco. The measurements were performed using 10x magnification and $1 \mathrm{x}$ field of view.

\subsubsection{Test procedure}

The ball on disc test device is used to generate friction data from a relatively broad range of operating conditions where one test cycle covers entrainment speeds between 0.34 to $9.6 \mathrm{~m} / \mathrm{s}$ and slide to roll ratios (SRR) from 0.0002 to 0.49 . Both ball and disc specimens were cleaned with heptane and ethyl alcohol before starting the experiments for each of the test cases. Before starting the experiments for each test case, the test device is warmed up to the desired operating temperature during approximately 60 minutes with oil circulation over both ball and disc to ensure temperature stability. When thermal stability is reached a 50 or $300 \mathrm{~N}$ load, equivalent to 1.07 or $1.94 \mathrm{GPa}$ maximum Hertzian pressure is applied and the machine is calibrated for pure rolling by adjusting spindle angle and positioning of the ball to ensure a condition of no spinning. These settings are then held constant for 20 minutes to ensure a mild run-in. Subsequently the test cycle is started that contains several loops where SRR is held constant for each loop and the entrainment speed is ramped from 9.6 to $0.34 \mathrm{~m} / \mathrm{s}$. In the first loop the SRR is held at 0.0002 and is then continuously increased with each loop until it reaches 0.49 . The same test cycle is repeated in the same track for both ball and disc until the absolute friction coefficient for each measured combination of entrainment speed and SRR does not differ more than 0.001 from the previous test cycle, excluding SRR below 0.0016 where the accuracy of the machine is slightly lower. When this occurs, the system is considered run in, and the data from the final test cycle is used for evaluation. The temperature of the oil bulk and fluid film at the disc surface is typically deviating less than $\pm 1.5^{\circ} \mathrm{C}$ from the target temperature of $40{ }^{\circ} \mathrm{C}$ during testing. The actual contact temperatures are however higher than the bulk oil temperature. In the most severe cases with high entrainment speed, SRR and coefficient of friction $(\mathrm{COF})$, the contact temperature will increase several tens of degrees [14].

Data from each test is processed separately, and a triangle based linear interpolation is used between the data points measured for specific SRR's and entrainment speeds. The results are presented as 3D friction maps, that are discussed in detail in previous work [16].

\section{Results and discussion}

In this section, the results from both experiments and numerical simulations are presented. The results include friction coefficient measurements and calculations, film thickness calculations and comparisons in percentage of the difference between experiments and numerical calculations. It also shows 


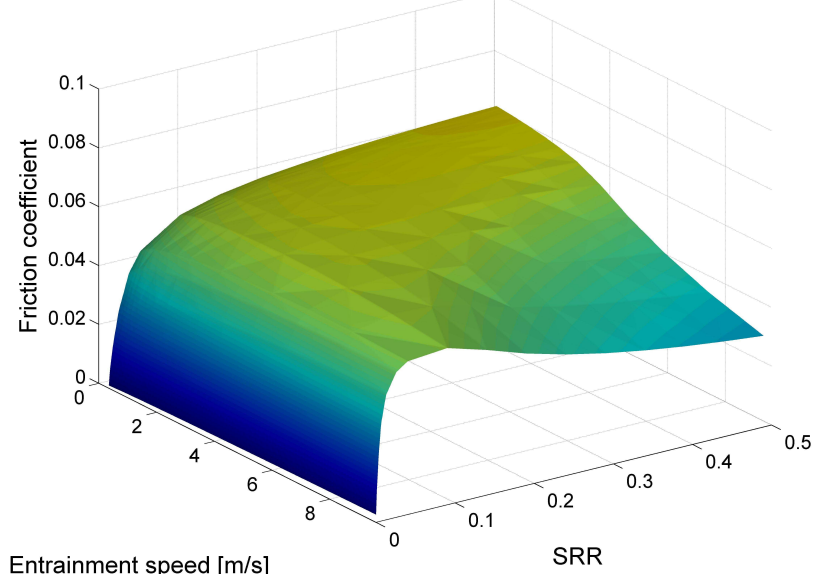

Entrainment speed [m/s]

(a) Simulation, $40^{\circ} \mathrm{C}, 1.07 \mathrm{GPa}$

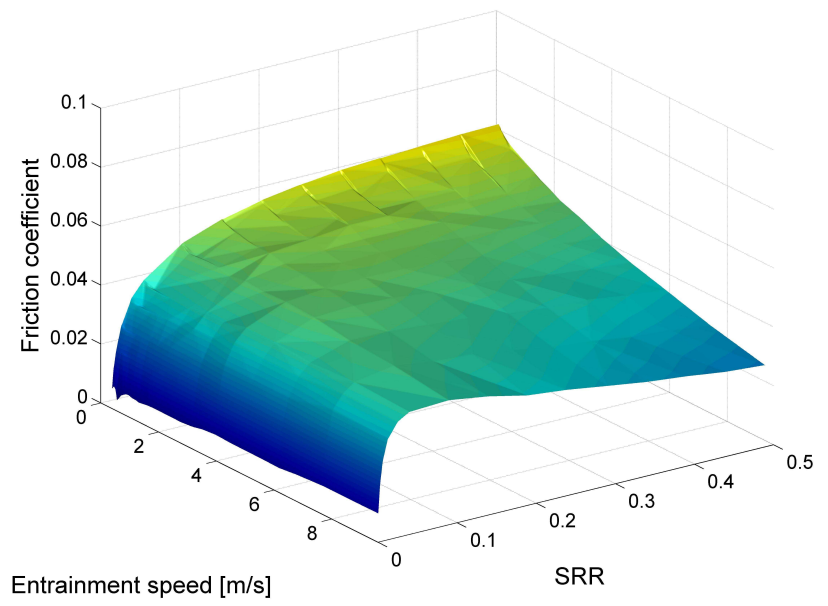

(c) Experiment, $40^{\circ} \mathrm{C}, 1.07 \mathrm{GPa}$

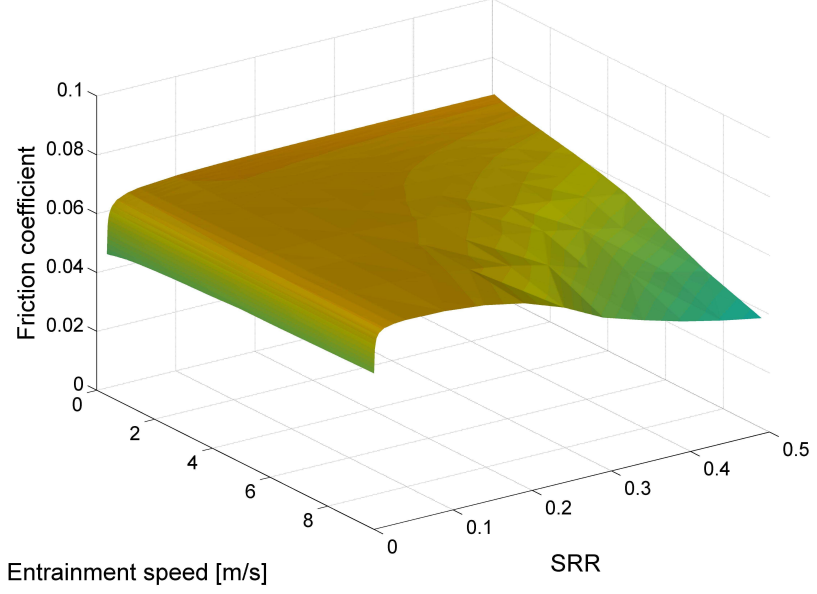

(b) Simulation, $40^{\circ} \mathrm{C}, 1.94 \mathrm{GPa}$

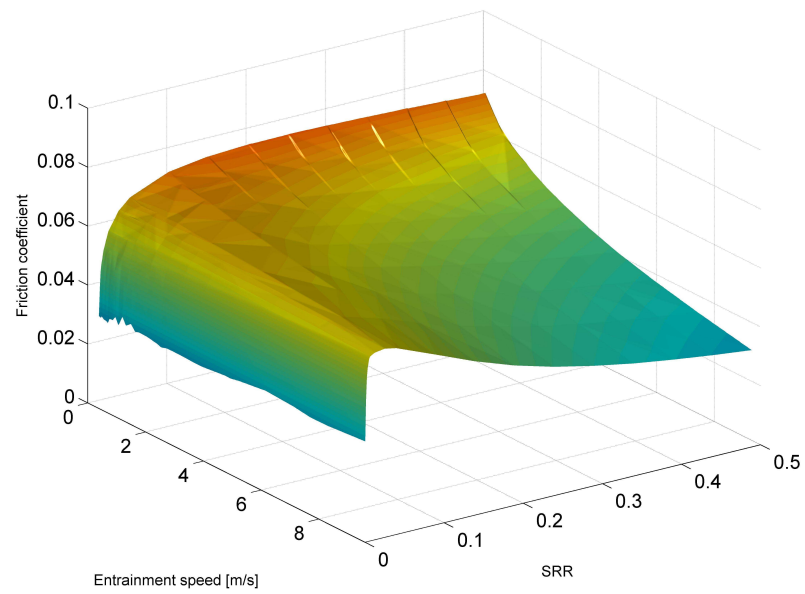

(d) Experiment, $40^{\circ} \mathrm{C}, 1.94 \mathrm{GPa}$

Figure 1: 3D friction maps - Comparison between experiment and calculation

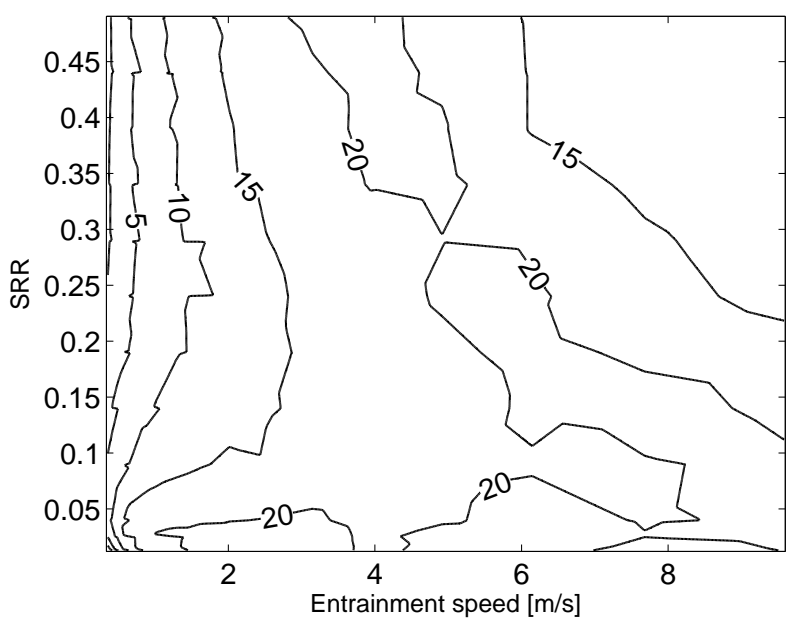

Figure 2: Difference in friction coefficient, Calculation-experiment [\%], 40 ${ }^{\circ} \mathrm{C}$, $1.07 \mathrm{GPa}$

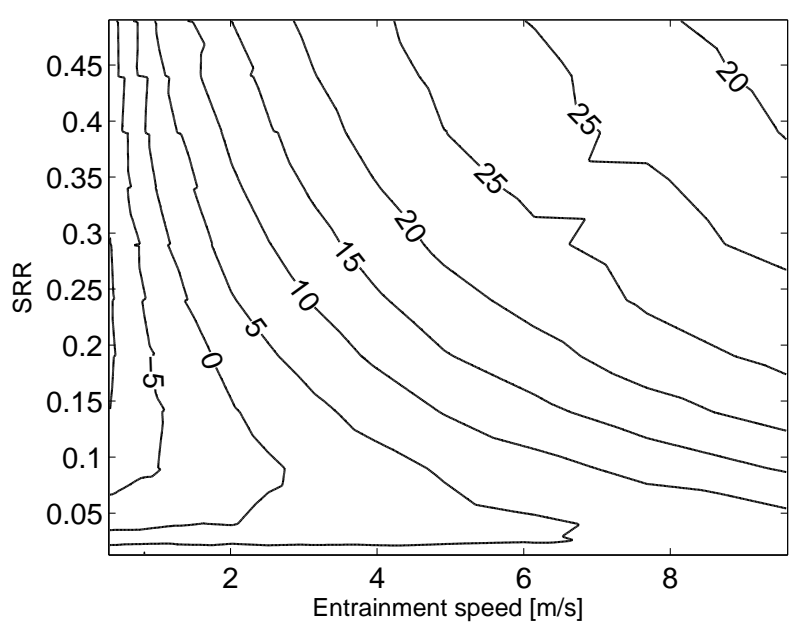

Figure 3: Difference in friction coefficient, Calculation-experiment [\%], $40^{\circ} \mathrm{C}$, $1.94 \mathrm{GPa}$ 


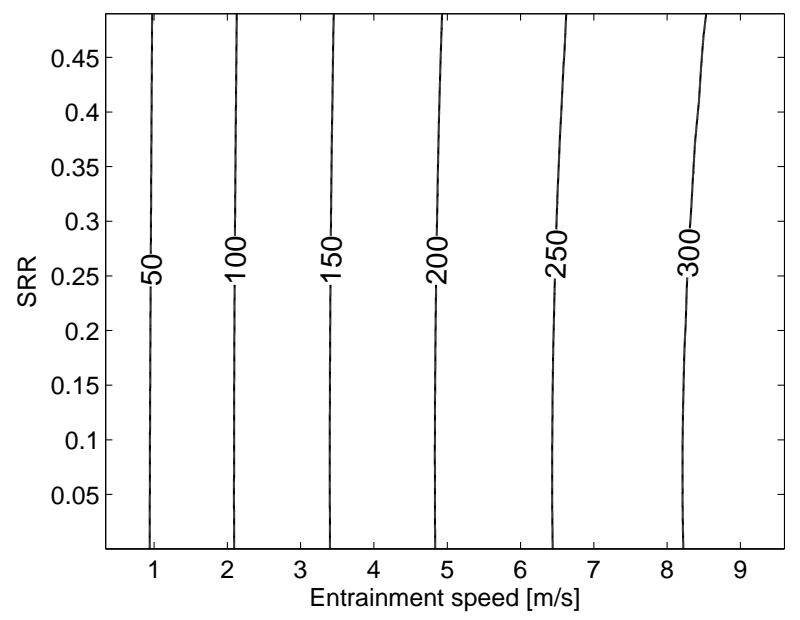

Figure 4: Calculated minimum film thickness [nm], 40 ${ }^{\circ} \mathrm{C}, 1.07 \mathrm{GPa}$

the calculated power generated from friction in the simulations for both pressures. The results from the calculations and the experiments can be seen in Fig. 1 where Figs. 1(a) and 1(b) are from calculations, and Figs. 1(c) and 1(d) are from experiments.

Figure 2 shows the difference between the experiment and calculation in percent for the test performed at $1.07 \mathrm{GPa}$, and the difference for the $1.94 \mathrm{GPa}$ test is shown in Fig. 3. A positive number in those figures indicates that the predicted coefficient of friction is higher than the experimentally obtained value, whereas a negative number indicates that the predicted friction coefficient is lower than the experimentally obtained value. The calculated minimum film thickness for the 1.07 and $1.94 \mathrm{GPa}$ cases for the entire region of the tests are shown in Figs. 4 and 5. Figs. 6 and 7 show the generated power from friction in the experiments for the 1.07 and $1.94 \mathrm{GPa}$ cases respectively. The friction power is calculated as: $W_{f}=\mu * U_{e} * S R R * L$. Where $\mu$ is the coefficient of friction, $U_{e}$ is the mean entrainment speed and $L$ is the applied load. The loads are 50 and 300 $\mathrm{N}$ respectively.

\subsection{Film thickness and roughness effects}

The minimum film thickness values presented in Figs. 4 and 5 are taken from the numerical calculations. It is worth noting that the film thickness, especially for the lower pressure case, Figure 4 are almost independent of SRR. The increased temperature in the inlet of the contact with increasing entrainment speeds and SRRs are not substantial enough to considerably influence the film thickness. For the higher pressure case, Fig. 5 the effect of increasing entrainment speed and SRRs on film thickness is larger, which is not surprising considering the difference in friction power, see Figs. 6 and 7. However, even here the effect on film thickness is small. The effect of thermal heating on friction is of much greater importance and will be discussed later.

Most likely the measurement performed at the lower contact pressure, 1.07 GPa, Fig 1(c), is in the full film regime at all

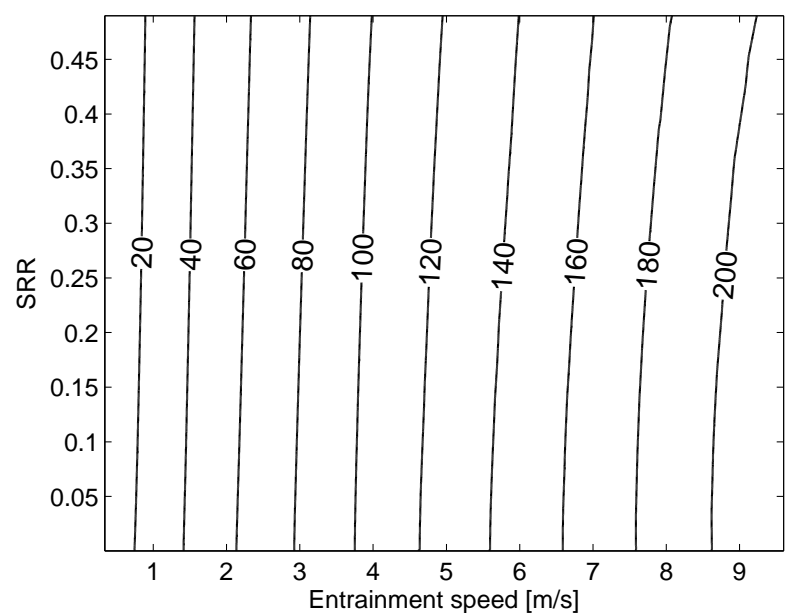

Figure 5: Calculated minimum film thickness $[\mathrm{nm}], 40^{\circ} \mathrm{C}, 1.94 \mathrm{GPa}$

combinations of speeds and SRRs, possibly with the exception of the lowest speeds. The calculated minimum film thickness for the lowest tested speeds at 1.07 GPa is in the range of 35-40 nm, as seen in Fig. 4, in comparison with a combined roughness (RMS) for the contacting surfaces of about $43 \mathrm{~nm}$. The possibility for slightly smoother surfaces after running in, with the combination of surface amplitude reduction $[17,18]$ makes full film lubrication possible. For the test performed at the higher contact pressure, $1.94 \mathrm{GPa}$, it is more likely that asperity interactions occurs at the lowest speeds, even considering running in effects and amplitude reduction. It should however be pointed out that the surface roughness measurements performed after the tests did not show any statistical reduction in RMS roughness indicating that any running in effects were very small. The calculated minimum film thickness for the higher pressure case can be seen in Fig. 5 .

\subsection{Friction}

As discussed in previous work [16] the friction maps could be divided into several different regimes where friction is governed and influenced by different factors. Recent work on friction regimes in quantitative EHL [11] has led to more detailed knowledge about the regimes, and the addition of one more regime is also included in the present work. The regions can be seen in Fig. 8, which results from a measurement conducted at the same conditions as Fig. 1(d) but with rougher surfaces for a more pronounced mixed lubrication region.

In the linear region, shear stress is proportional to shear rate, and will thus lead to a linear increase in friction. This linear relationship is only seen at low SRRs, and the upper limit (in SRR) becomes even lower with increasing pressures as the shear stress increases faster with shear rate at higher pressures and at lower entainment speeds.

The non-linear region is influenced by shear thinning and dominated by the growth of a limiting stress region. The shear stress will no longer increase proportional to shear rate. It will rather increase at a reduced rate until limiting shear stress is at- 


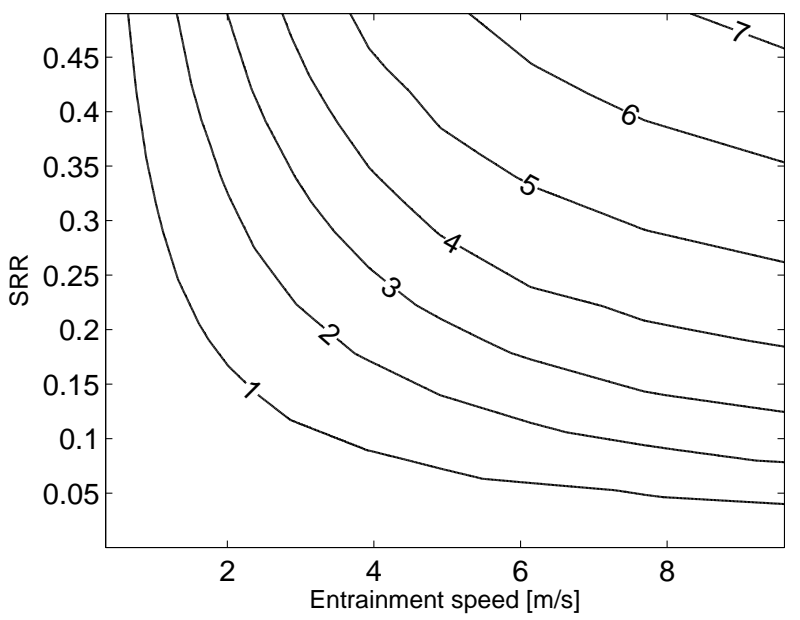

Figure 6: Friction power [W], $40^{\circ} \mathrm{C}, 1.07 \mathrm{GPa}$

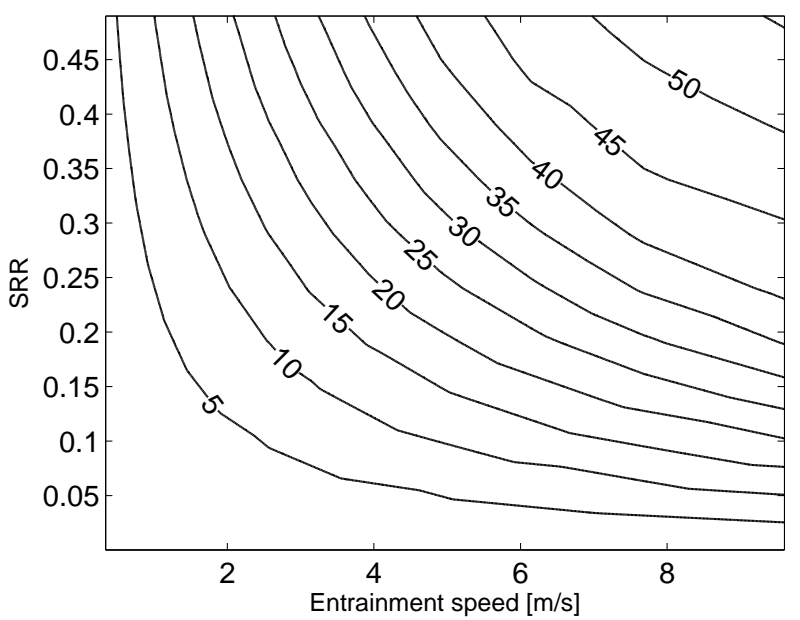

Figure 7: Friction power [W], $40^{\circ} \mathrm{C}, 1.94 \mathrm{GPa}$

tained over much of the contact. Then, shear stress becomes independent of shear rate. However, if one keeps on increasing the sliding speed, thermal effects eventually dominate the frictional response overwhelming other effects including limiting shear stress which is no longer reached. As a consequence, friction starts decreasing with increasing SRR due to combined thermal and shear-thinning effects. This regime has been identified as the Thermoviscous regime.

In the boundary between the mixed lubrication regime and the Thermoviscous regime lies the Plateau regime. Here, friction reaches an asymptotic value and shows little variation indicating that the frictional response of the contact is governed by the limiting shear stress behavior of the lubricant.

The last region is the mixed lubrication region where asperity contacts occur between the surfaces, and the coefficient of friction is therefore a combination of hydrodynamic effects and asperity interaction.

By looking at the overview of the four cases in Figure 1 it is clear that the numerical model manages to capture the overall

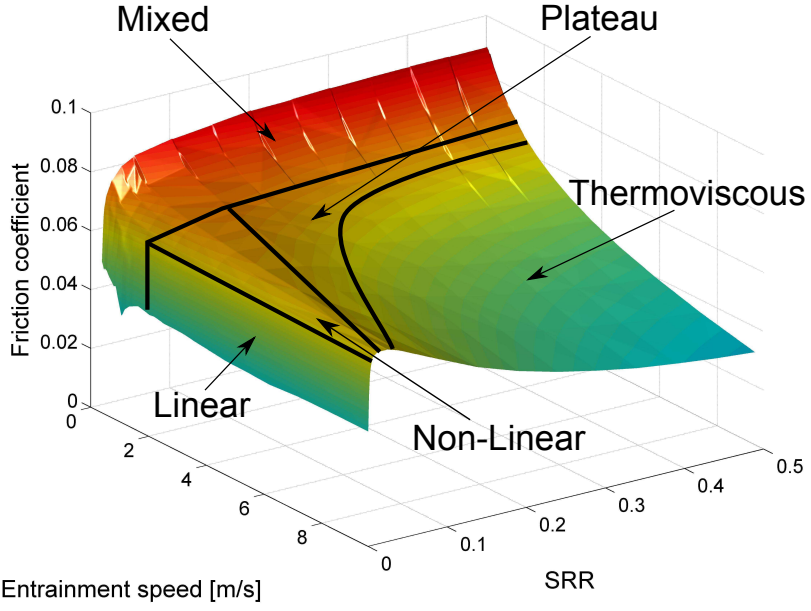

Figure 8: 3D friction map with regimes

frictional behavior such as the linear increase in friction with shear rate at low SRR, the non linear increase in friction leading to the limiting shear stress and the reduction of friction at higher entrainment speeds and SRRs due to thermal effects. Mixed lubrication effects are however not included in the numerical model, and could therefore be part of the difference between the numerical prediction and the experimental results at the lowest entrainment speeds, as discussed in previous section.

At lower entrainment speeds, up to about $2 \mathrm{~m} / \mathrm{s}$ the predictions are in general not far of, the deviation is typically below $10 \%$ and in line with previous comparisons at these entrainment speeds [9]. At higher speeds the coefficient of friction is constantly overestimated by the numerical model, and generally more so at higher SRRs. When looking at absolute values, and the difference between the numerical calculations and the experiment as presented in Figs. 2 and 3 it is clear that the model does not accurately predict all parts of the tested region, or that some of the boundary conditions in the numerical model does not fit the experiment.

One of the most striking differences between the predictions and the experiments are the plateu regime in the predictions, Figs. 1(a) and 1(b) where the coefficient of friction is almost constant at a high level over a wide range of entrainment speeds and SRRs. Such behavior is not as distinct in the experiments, Figs. 1(c) and 1(d) where instead the friction coefficients drops faster with an increase in entrainment speeds and SRRs.

By looking at the difference in $\%$ between the predictions and the experiments in Figs. 2 and 3 it is clear that the levels of difference are situated in the same manner as the levels of friction power in Figs. 6 and 7. It is therefore likely that one of the main reasons for the deviation between the prediction and the experiments are found in how the model handles thermal effects and/or some thermal behavior in the test rig and specimens that are not matched in the boundary conditions in the numerical model. It should however be pointed out that the biggest discrepancies does not occur at the highest entrainment speeds and SRRs, but rather in connection to the plateau regime. 
The numerical model is using bulk temperature as a boundary condition for the inlet temperature of the lubricant and a steady state condition is calculated for the specific running conditions (entrainment speed and SRR.) Any global effects on the specimen temperature are therefore not taken into account, while in the experiment it is possible that especially the ball surface temperature is increasing during the test. As a specific running condition is measured in the test rig it takes a few seconds for the machine to adjust rotational speeds of both specimens to achieve the correct entrainment speed and SRR. Some additional time is also required for the data capture process. It is possible that during this time, the specimens are heated due to the friction generated in the contact, and therefore the lubricant temperature will be slightly higher in the inlet and central region of the contact compared to the numerical model. Looking at the friction power for the low pressure, 1.07 GPa case in Fig. 6 , there is a maximum of $7 \mathrm{~W}$. For the high pressure, $1.94 \mathrm{GPa}$ case in Fig. 7, with a maximum friction power of $55 \mathrm{~W}$, this is more likely to have an effect on the results, and may be the reason why the deviation in general is slightly larger for the higher pressure case at higher sliding speeds. If the reasoning above is the cause for the largest part of the deviations, it is not sufficient to consider an ideal circular contact. Rather must the effect of the global geometry on intake and surface temperature be taken into account.

However, it is also possible that the deviations between the experimental results and the predictions are due to shortcomings in the rheology models used in the study.

As previously discussed, recent research [11] suggests that it is not possible to accurately estimate the limiting shear stress coefficient from EHL friction measurements, since shearthinning was found to affect the friction coefficient even when the limiting shear stress is reached in parts of the contact.

Moreover is the limiting shear stress in the numerical model used in this work assumed to be only depending on pressure. It has been shown earlier in measurements that temperature has an influence on the limiting shear stress [19]. It is reasonable to believe that this is one of the reasons why the plateu regime is more pronounced in the numerical predictions. With the inclusion of the temperature dependence of limiting shear stress the plateu would probably level off with increased sliding due to a reduction in limiting shear stress before thermal effects starts to dominate the friction behavior.

Finally, even rather small deviations in the Carreau equation and Vogel like function will have a substantial impact on the resulting friction coefficient.

\section{Conclusion}

In this paper the results from a true friction prediction investigation are shown. The transport properties for the fluid squalane was measured at one laboratory, and was later used at a second laboratory to predict friction in a numerical model for a circular EHL contact. Finally the same parameters were used for friction measurements in a ball on disc machine in a third laboratory. The investigated conditions includes a combination of higher entrainment speeds and pressures than previously reported in this kind of investigation. No tuning of the lubricant properties, model and test setup was applied.

The predictions were found to capture the main features of the friction behavior at different entrainment speeds and SRRs. In absolute values the numerical results were found to be remarkably good considering the true prediction approach. At low entrainment speeds the deviations between the numerical prediction and the experimental results are in general less than $10 \%$, while increasing at higher entrainment speeds and SRRs. The authors believe that to achieve even better precision in the predictions there is a need to refine the assessment of the limiting shear stress of the lubricant, including the effect of temperature. In addition may it be necessary to refine the boundary conditions in the model to account for global effects on temperature.

The use of primary measurements or "true" measurements of the liquid properties in EHL has distinct advantages over the previous techniques of adjusting properties to satisfy perhaps inappropriate assumptions. If friction is to be controlled by lubricant selection and formulation, there must be an understanding of the contribution of each of the transport properties to the energy dissipation. An example can be made for the liquid in this study, squalane. Johnston et al. [20] adjusted the pressureviscosity coefficient of squalane to make the classical formulas agree with measured film thickness. If this coefficient, which is about one-half of the value obtained in viscometers, had been used to extrapolate viscosity to the full pressure of the contact in the conventional manner, the importance of the low-shear viscosity would have been severely understated and a more severe form of shear-thinning would have to be invoked to explain the friction. However, the use of the true prediction approach investigated in this article will generally require more effort from a measurement and modeling point of view compared to previous techniques.

\section{Acknowledgement}

The authors wish to thank Ove Andersson at Umeå University for performing the thermal properties measurements. The authors from Luleå wish to thank Swedish Foundation for Strategic Research (ProViking) for financial support. Bair was supported by the Center for Compact and Efficient Fluid Power, a National Science Foundation Engineering Research Center funded under cooperative agreement number EEC-0540834.

\section{References}

[1] Bridgman, P.W.. The Physics of High Pressure. G. Bell and sons, ltd, London; 1931, p. 98-147, 295-356.

[2] Kleinschmidt, R.V., Bradbury, D., Mark, M.. Viscosity and density of over forty lubricating fluids of known composition at pressures to 150,000 psi and temperatures to 425 f. Tech. Rep.; ASME, New York; 1953.

[3] Hutton, J.F.. Theory of rheology. Interdisciplinary Approach to Liquid Lubricant Technology (PM Ku, Ed) 1973;NASA, Washington:187-261.

[4] Bair, S., McCabe, C., Cummings, P.T.. Calculation of viscous ehl traction for squalane using molecular simulation and rheometry. Tribology Letters 2002;13(4):251-254. doi:10.1023/A:1021011225316. 
[5] Bair, S., Vergne, P., Querry, M.. A unified shear-thinning treatment of both film thickness and traction in ehd. Tribology Letters 2005;18(2):145-152. doi:10.1007/s11249-004-1770-y.

[6] Liu, Y., Wang, Q.J., Bair, S., Vergne, P.. A quantitative solution for the full shear-thinning EHL point contact problem including traction. Tribology Letters 2007;28(2):171-181. doi:10.1007/s11249-007-9262-5.

[7] Habchi, W., Demici, I., Eyheramendy, D., Morales-Espejel, G., Vergne, P.. A finite element approach of thin film lubrication in circular ehd contacts. Tribology International 2007;40(10-12):1466-1473. doi:10.1016/j.triboint.2007.01.017.

[8] Habchi, W., Eyheramendy, D., Vergne, P., Morales-Espejel, G. A full-system approach of the elastohydrodynamic line/point contact problem. ASME Journal of Tribology 2008;130,021501:1-10. doi:10.1115/1.2842246.

[9] Habchi, W., Vergne, P., Bair, S., Andersson, O., Eyheramendy, D., Morales-Espejel, G.E.. Influence of pressure and temperature dependence of thermal properties of a lubricant on the behaviour of circular tehd contacts. Tribology International 2010;43(10):1842-1850. doi:10.1016/j.triboint.2009.10.002.

[10] Bair, S., Winer, W.O.. A new high-pressure, high-shear stress viscometer and results for lubricants. STLE 1993;36(4):721-725.

[11] Habchi, W., Bair, S., Vergne, P.. On friction regimes in quantitative elastohydrodynamics. Tribology I 2013;58:107-117. doi:10.1016/j.triboint.2012.10.005.

[12] Bair, S.. Reference liquids for quantitative elastohydrodynamics. Tribology Letters 2006;22(2):197-206. doi:10.1007/s11249-006-9083-y.

[13] Bair, S., McCabe, C., Cummings, P.T.. Comparison of nonequilibrium molecular dynamics with experimental measurements in the nonlinear shear-thinning regime. Physical Review Letters 2002;5(4):583021583024. doi:10.1103/PhysRevLett.88.058302.

[14] Habchi, W., Eyheramendy, D., Bair, S., Vergne, P., MoralesEspejel, G.. Thermal elastohydrodynamic lubrication of point contacts using a newtonian/generalized newtonian lubricant. Tribology letters 2008;30(1):41-52. doi:10.1007/s11249-008-9310-9.

[15] Yang, P., Wen, S.. A generalized reynolds equation for nonnewtonian thermal elastohydrodynamic lubrication. Journal of Tribology 1990;112(4):631-636.

[16] Björling, M., Larsson, R., Marklund, P., Kassfeldt, E.. Elastohydrodynamic lubrication friction mapping - the influence of lubricant, roughness, speed, and slide-to-roll ratio. Proceedings of the Institution of Mechanical Engineers, Part J: Journal of Engineering Tribology 2011;225(7):671681. doi:10.1177/1350650111403363.

[17] Lubrecht, A.A., Venner, C.H.. Elastohydrodynamic lubrication of rough surfaces. Proceedings of the Institution of Mechanical Engineers, Part J: Journal of Engineering Tribology 1999;213:397-403.

[18] Venner, C., Lubrecht, A.. An engineering tool for the quantitative prediction of general roughness deformation in EHL contacts based on harmonic waviness attenuation. Proceedings of the Institution of Mechanical Engineers, Part J: Journal of Engineering Tribology 2005;219(5):303312. doi:10.1243/135065005X33973.

[19] Höglund, E.. Influence of lubricant properties on elastohydrodynamic lubrication. Wear 1999;232(2):176-184. doi:10.1016/S00431648(99)00143-X.

[20] Johnston, G., Wayte, R., Spikes, H.. The measurement and study of very thin lubricant films in concentrated contacts. Tribology Transactions 1991;34(2):187-194. 